\title{
In Vitro Antibacterial Activity of Curcumin-Polymyxin B Combinations against Multidrug-Resistant Bacteria Associated with Traumatic Wound Infections
}

\author{
Jonathan W. Betts, ${ }^{* \dagger}{ }^{\dagger}$ Amir S. Sharili, ${ }^{\ddagger}$ Roberto M. La Ragione, ${ }^{\dagger}$ and David W. Wareham ${ }^{\S}$ \\ ${ }^{\dagger}$ School of Veterinary Medicine, Faculty of Health and Medical Sciences, University of Surrey, Guildford GU2 7AL, U.K. \\ ${ }^{\ddagger}$ Cell Biology and Cutaneous Research and ${ }^{\S}$ Antimicrobial Research Group, Blizard Institute, Barts \& The London School of \\ Medicine and Dentistry, Queen Mary University of London, London E1 2AT, U.K.
}

ABSTRACT: Bacterial infections resulting from nonsurgical traumatic wounds can be life threatening, especially those caused by multidrug-resistant (MDR) bacteria with limited therapeutic options. The antimicrobial activity of polymyxin B (1) and curcumin (2) alone and in combination was determined versus MDR bacterial isolates associated with traumatic wound infections. Cytotoxicity assays for $\mathbf{1}$ and $\mathbf{2}$ were undertaken in keratinocyte cell lines. Minimum inhibitory concentrations of 1 were significantly reduced in the presence of 2 (3- to 10-fold reduction), with synergy observed. Timekill assays showed the combinations produced bactericidal activity. Cytotoxicity assays indicate the toxicity of 2 was reduced in the presence of 1 .

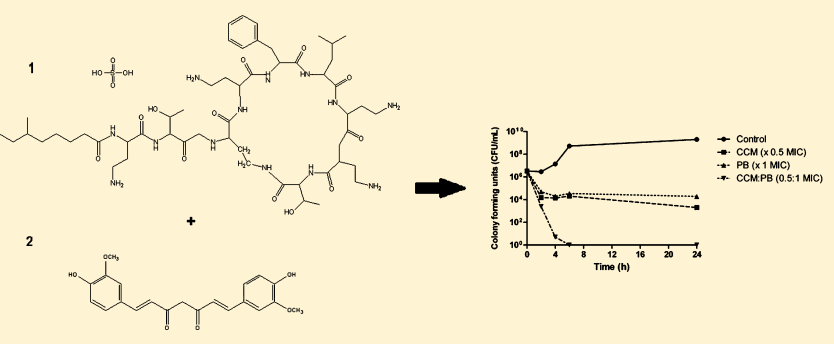

C omplicated bacterial infections resulting from nonsurgical traumatic wounds and/or burns arise rapidly due to the loss/penetration of the natural barrier, the skin, and can be life threatening. These infections are commonly caused by bacteria such as Staphylococcus aureus, Streptococcus pyogenes, vancomycin-resistant Enterococci, Pseudomonas aeruginosa, and Escherichia coli. ${ }^{1}$ An increasing number of these infections are caused by multidrug-resistant (MDR) strains, which can lead to longer hospital stays, increased financial burden, and higher rates of morbidity and mortality. ${ }^{2}$ Multidrug resistance has led to the use of "last line" antibiotics such as carbapenems or older antibiotics such as polymyxins, which can produce renal toxicity. ${ }^{3}$ Increased resistance to carbapenems is also increasing due to emergence and rapid dissemination of carbapenemasesproducing strains. ${ }^{4}$ Several studies report limited therapeutic options to treat MDR bacterial infections. ${ }^{5-7}$ Physicians often use unorthodox antibiotic combinations to increase the efficacy of treatment. ${ }^{8}$

Like colistin, $\mathbf{1}$ is a cyclic polypeptide antibiotic, with antibacterial activity against Gram-negative bacteria such as Acinetobacter baumannii, P. aeruginosa, E. coli, and Klebsiella pneumoniae and has been used topically for many years for the treatment of skin infections. In 1956, it was used in combination with B-bacitracin-neomycin as an effective antimicrobial ointment against a variety of micro-organisms. ${ }^{9}$ 1 has also been used in selective decontamination regimes to suppress the growth of Enterobacteriaceae. ${ }^{10}$ Like colistin (polymyxin E), 1 has seen increased use to treat Gram-negative bacterial infections, due to the emergence of resistance to antibiotics such as carbapenems. ${ }^{11}$ However, increased resistance to polymyxins has emerged, ${ }^{12}$ which is likely due to their revival in clinical settings and continued agricultural use. The recent discovery of the mobile colistin resistance gene (MCR-1) in humans and animals has increased the concern of the future efficacy of polymyxins. ${ }^{13}$

One possible solution would be to use 1 in combination with the phenolic compound curcumin (2). 2 is a major component of the spice turmeric from the plant Curcuma longa Linn. and has been used as a natural antimicrobial in Asia for many years. Previous work has shown 2 to be an effective antimicrobial agent against various human pathogens. ${ }^{14,15}$ It has also been previously recognized for its ability to prevent oxidative stress in skin cells and for its potential to aid in wound healing. ${ }^{16}$ Moreover, studies have reported the ability of $\mathbf{2}$ to reduce epidermal tumor size and formation in mice. ${ }^{17}$ Combinations of 2 with other antimicrobials has also been shown to be effective against MDR bacteria such as $A$. baumannii. ${ }^{18}$ Novel combination therapy has recently been proposed for the treatment of complicated skin infections caused by multidrugresistant bacteria using antibiotics or antibiotics in combination with natural compounds. ${ }^{19}$ Using combination therapy, the efficacy of drugs such as the polymyxins could be preserved and/or extended.

Here we aimed to determine the in vitro activity of polymyxin B and curcumin, alone and in combination, against antibiotic-susceptible and -resistant Gram-positive (Enterococci, S. aureus, and Streptococci) and Gram-negative (A. baumannii, E. coli, $P$. aeruginosa, and $S$. maltophilia) bacterial isolates associated with traumatic wound infections.

Received: April 18, 2016

Published: June 13, 2016 
Table 1. Minimum Inhibitory Concentrations (MICs) of Polymyxin B (PB) and Curcumin (CCM) Alone or in Combination and Fractional Inhibitory Concentration Indices (FICIs) for Important Potential Pathogens of Traumatic Wounds Expressing a Variety of Antimicrobial Resistance Mechanisms ${ }^{a}$

\begin{tabular}{|c|c|c|c|c|c|c|}
\hline \multirow[b]{2}{*}{ isolate } & \multirow[b]{2}{*}{ characteristics } & \multicolumn{2}{|c|}{ MICs $(\mu \mathrm{M})$} & \multicolumn{2}{|c|}{ MICs in combination } & \multirow[b]{2}{*}{ FICI } \\
\hline & & 1 & 2 & $1+2$ & $2+1$ & \\
\hline E. faecium OEF42 & VRE (vanA) & 98 & 350 & 3.1 & 22 & 0.094 \\
\hline E. faecium OEF65 & $\operatorname{VRE}(\operatorname{van} A / B)$ & 49 & 174 & 1.5 & 43 & 0.281 \\
\hline E. faecalis ATCC 29212 & type strain & 98 & 350 & 0.096 & 87 & 0.251 \\
\hline S. aureus $\mathrm{Mu} 50$ & VISA (vraSR) & 98 & 2780 & 1.5 & 43 & 0.047 \\
\hline S. aureus ATCC 25923 & type strain & 98 & 2780 & 6.1 & 87 & 0.156 \\
\hline S. aureus NCTC 12493 & MRSA (mecA) & 98 & 2780 & 0.79 & 43 & 0.031 \\
\hline S. pyogenes SPY1 & wound isolate & 6.1 & 350 & 0.19 & 43 & 0.094 \\
\hline S. pyogenes SPY2 & wound isolate & 12.3 & 174 & 0.79 & 22 & 0.188 \\
\hline S. pyogenes SPY3 & wound isolate & 3.1 & 174 & 0.79 & 43 & 0.500 \\
\hline E. coli $\mathrm{EC} 2$ & CTX-M-15 & 0.096 & 2780 & 0.024 & 43 & 0.266 \\
\hline E. coli EC204 & NDM-1 & 0.096 & 2780 & 0.024 & 174 & 0.313 \\
\hline E. coli NCTC 12241 & type strain & 0.048 & 2780 & 0.024 & 43 & 0.520 \\
\hline A. baumannii $\mathrm{AB} 12$ & OXA-23 & 0.19 & 2780 & 0.024 & 87 & 0.156 \\
\hline A. baumannii $\mathrm{AB} 14$ & OXA-23 clone 1 & 0.096 & 1390 & 0.024 & 174 & 0.375 \\
\hline A. baumannii $\mathrm{AB} 16$ & OXA-23 clone 2 & 0.19 & 2780 & 0.012 & 22 & 0.068 \\
\hline A. baumannii NCTC 19606 & type strain & 0.19 & 2780 & 0.012 & 22 & 0.068 \\
\hline P. aeruginosa PA01 & reference strain & 0.78 & 1390 & 0.012 & 43 & 0.031 \\
\hline P. aeruginosa PA14 & reference strain & 0.78 & 1390 & 0.012 & 22 & 0.023 \\
\hline$P$. aeruginosa $\mathrm{PA} 30$ & VIM-2 & 0.39 & 2780 & 0.19 & 174 & 0.563 \\
\hline P. aeruginosa ATCC 27853 & type strain & 0.39 & 1390 & 0.006 & 174 & 0.079 \\
\hline S. maltophilia SMS01 & L1, TEM & 6.1 & 2780 & 0.024 & 22 & 0.012 \\
\hline S. maltophilia SMB07 & L1, TEM & 1.5 & 2780 & 0.0313 & 22 & 0.023 \\
\hline S. maltophilia NCTC 10258 & type strain & 1.5 & 2780 & 0.008 & 22 & 0.012 \\
\hline
\end{tabular}

${ }^{a}$ MRSA, methicillin-resistant Staphylococcus aureus; VISA, vancomycin-intermediate resistant S. aureus; VRE, vancomycin-resistant Enterococcus; PA14, common, highly virulent clone. All $S$. pyogenes strains are antibiotic-susceptible wound isolates.
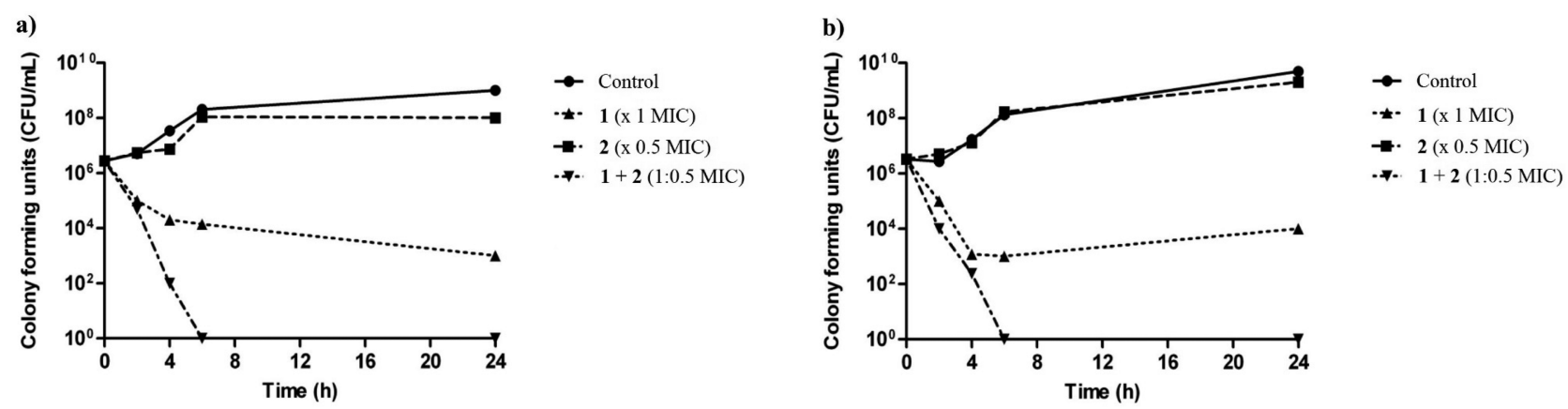

c)

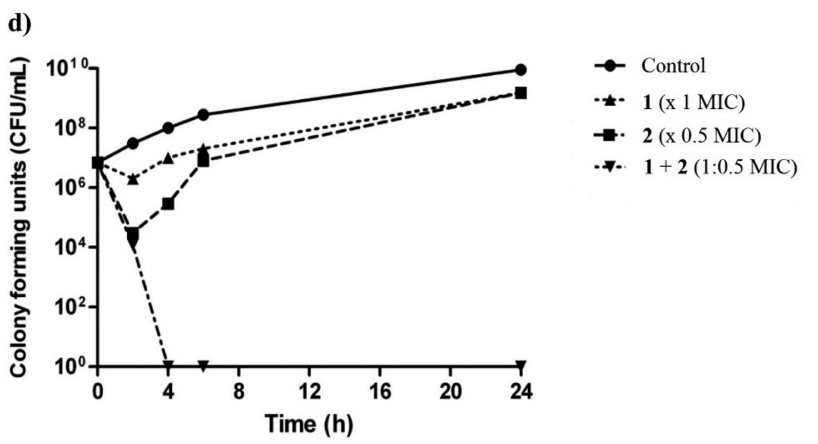

Figure 1. Time-kill curves of polymyxin B (PB) and curcumin (CCM) alone and in combination versus (a) A. baumannii (ATCC 19606), (b) S. maltophilia (NCTC 10258), (c) P. aeruginosa (ATCC 27853), and (d) Methicillin-resistant S. aureus (NCTC 12493).

1 produced good antibacterial activity against Gram-negative strains with minimum inhibitory concentrations (MICs) of 0.096-6.1 $\mu \mathrm{M}$ (Table 1). Antimicrobial activity was also demonstrated against isolates of S. pyogenes with MICs of 3-
$12.3 \mu \mathrm{M}$. As expected, very high MICs of $174-347 \mu \mathrm{M}$ for 1 were observed versus $S$. aureus and Enterococcus spp. and would normally be classed as unsusceptible. Compound 2 produced weak antibacterial activity with high MICs (174-2780 $\mu \mathrm{M})$ in 
all clinical isolates and type strains. When added in combination, $\mathbf{1}$ and $\mathbf{2}$ produced increased antibacterial activity with MICs significantly reduced (3- to 10 -fold reduction, $p<$ 0.005). The fractional inhibitory concentration indices (FICIs) calculated suggest that synergy was produced in $21 / 23$ strains $($ FICI $\leq 0.5)$. In $2 / 23$ strains an additive effect was produced (FICI > 0.5-4.0).

Time-kill results demonstrated that $\mathbf{2}$ was only effective against $P$. aeruginosa (ATCC 27853), with a $2 \log$ reduction in colony-forming units (CFU)/mL over $24 \mathrm{~h}$ (Figure 1d). Regrowth was observed for the three other strains over $24 \mathrm{~h}$, indicating that, alone, $\mathbf{2}$ was ineffective as an antibacterial agent. 1 was effective in reducing the $\mathrm{CFU} / \mathrm{mL}$ in the Gram-negative isolates by 2 logs at $6 \mathrm{~h}$. Regrowth was observed at $24 \mathrm{~h}$ in $S$. maltophilia (NCTC 10258). As expected 1 alone had no effect against MRSA (NCTC 12493), with growth almost equal to that of the no-drug control. However, the combination of 2 with 1 showed significant antibacterial activity, and complete cell death was observed in all strains between 4 and $6 \mathrm{~h}$ (Figure $1)$. The combination was clearly bactericidal, and significant synergy was produced, indicated by a $>3 \log \mathrm{CFU} / \mathrm{mL}$ difference between the agents used in combination versus alone. $^{18}$

The data presented here with the exception of $P$. aeruginosa agree with previous results, whereby 2 alone showed no antibacterial activity over $24 \mathrm{~h}$, in time-kill assays. ${ }^{18}$ This could be due to the insolubility of $\mathbf{2}$ or the inability of the compound to pass through the outer cell membrane/cell wall to reach its target.

Previous studies performed with B. subtilis indicate that the mode of antibacterial action of $\mathbf{2}$ involves the disruption of FtsZ protofilament activity, previously shown to be critical in bacterial cytokinesis. ${ }^{20}$ This disruption inhibits bacterial cell proliferation by inhibiting the assembly of the $\mathrm{Z}$ ring. 2 has been shown to inhibit the bacterial surface protein sortase $A$, preventing cell adhesion to fibronectin, leading to bacterial cell death in S. aureus. $^{21}$ The mechanism through which 2 nanoparticles (NPs) are believed to generate antibacterial action is by attaching to the bacterial cell wall, leading to disruption and eventual diffusion of NPs within the cell, resulting in further disrupting of biochemical processes. ${ }^{22}$

The addition of $\mathbf{1}$, a known membrane permeabilizer, may result in greater quantities of $\mathbf{2}$ entering Gram-negative bacterial cells, leading to increased toxicity. However, increased toxicity of the $\mathbf{1} / \mathbf{2}$ combination was also observed against Gram-positive bacteria. This in part could be due to the ability of $\mathbf{2}$ to cause bacterial cell wall damage, ${ }^{23}$ allowing $\mathbf{1}$ to disrupt the physical integrity of phospholipid bilayer of the inner membrane. $^{24}$

Cytotoxicity of 1 alone was very low $(2 \% \pm 0.01$ percentage point [pp] at $6.1 \mu \mathrm{M})$, whereas 2 showed $34 \%( \pm 2 \mathrm{pp})$ toxicity at concentrations of $174 \mu \mathrm{M}$ (Figure 2). However, the cytotoxicity of $\mathbf{2}$ versus $\mathrm{HaCaT}$ keratinocyte cells was reduced significantly $(22 \% \pm 3 \mathrm{pp}, p=<0.01)$ when used in combination with 1 , at concentrations of $43.4: 1.5 \mu \mathrm{M}$. Although cytotoxicity in combination is reduced in keratinocytes, it is increased versus bacterial cells, an important prerequisite for a topical therapy.

Although previous studies have also shown that $\mathbf{2}$ induces apoptosis in epidermal cells at $12.5-25 \mu \mathrm{M},{ }^{25}$ other studies have demonstrated that greater concentrations of $\mathbf{2}$ are required to induce in vitro cytotoxicity, with $\geq 25 \mu \mathrm{M} 2$ required for a $50 \%$ reduction in $\mathrm{HaCaT}$ (keratinocyte) cells and $\geq 50 \mu \mathrm{M}$
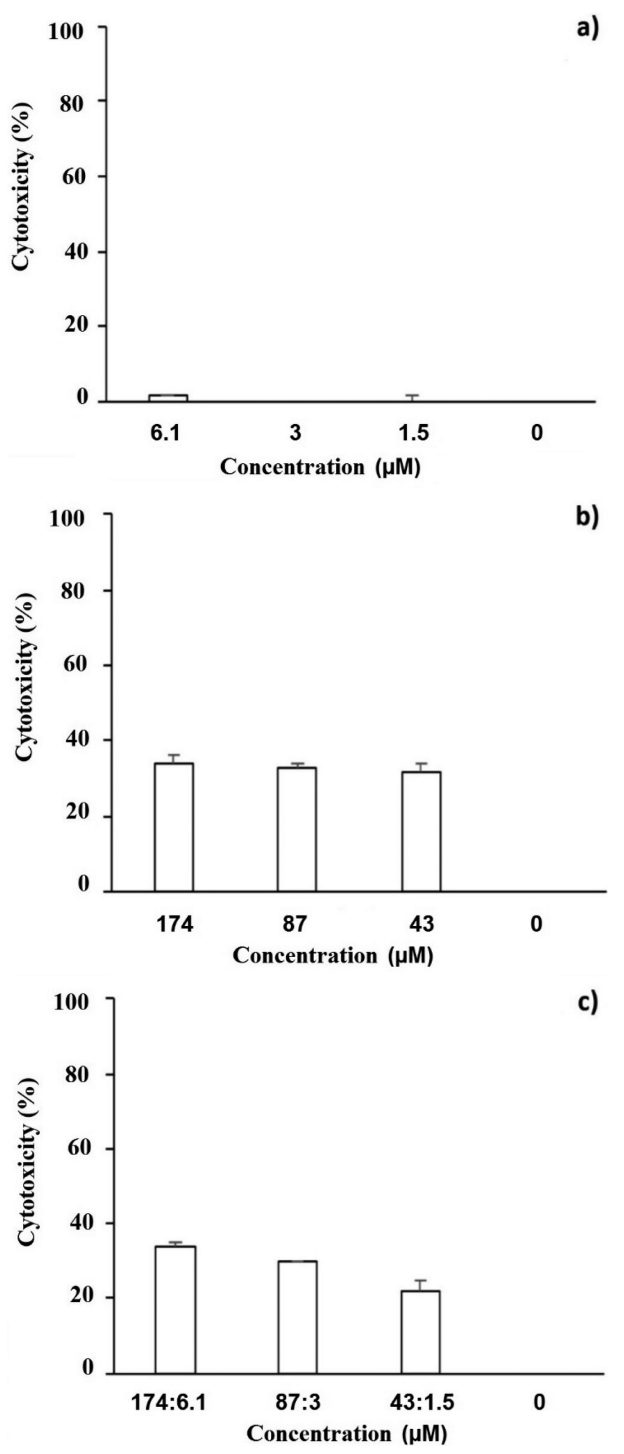

Figure 2. Cytotoxicity via lactase dehydrogenase assay of (a) polymyxin B (1), (b) curcumin (2), and (c) combinations of both agents in $\mathrm{HaCaT}$ cells \pm standard deviation, with the combination $(43: 1.5 \mu \mathrm{M})$ showing less toxicity than 2 alone $(p=<0.01)$.

required for a $50 \%$ reduction in melanocytes. ${ }^{26}$ These would be equal or higher than the concentrations of 2 required in combination to inhibit the growth of $70 \%$ of the isolates tested in this study. Although 2 exposure in vitro caused cytotoxicity in $\mathrm{HaCaT}$ keratinocytes in this study, previous topical applications have shown no skin irritation in a live rat model, ${ }^{27}$ indicating whole skin models have increased resistance to toxicity from 2 .

Data from phase I clinical trials have also shown that $\mathbf{2}$ is not toxic to humans up to concentrations of $21.72 \mathrm{mM}$, when taken orally. $^{28,29}$ A randomized, double-blind, placebo-controlled clinical trial of the effectiveness of 2 to treat mastitis also found no skin toxicity at $8 \mathrm{~h}$ doses of $5.49 \mathrm{mM}$ applications. ${ }^{30}$ The trial also observed significant reduction in inflammation and pain, indicating topical therapies of $\mathbf{2}$ were successful in treating mastitis.

Previously research has suggested that in vitro deposition of 2 on the skin was low 24 h after topical application, ${ }^{31}$ which could be due to solubility issues of 2 . It was suggested that enhancers were required for effective topical applications of $\mathbf{2}$, if 
the desired therapeutic effects were to be achieved. Other natural compounds such as terpenes or polyphenols such as epigallocatechin gallate (EGCG) could be used to aid 2 penetration into the skin. ${ }^{31}$ Combinations of 2 and EGCG have previously shown potential as an antimicrobial therapy and could be used as a third agent with $\mathbf{1}$ and $2 .^{18}$ Another option would be to use $\mathbf{2}$ nanoparticles in the formulation. 2 nanoparticles have previously shown to have enhanced antibacterial activity, water solubility, and a reduced risk of cytotoxicity in human cells. $^{22}$

In conclusion, antimicrobial synergy produced between 1 and 2 suggests that combinations of the two compounds could be used clinically for topical therapy to treat or prevent traumatic wound infections of the skin. The addition of 2 to current treatment containing 1 would not only increase the spectrum of activity to include Gram-positive bacteria but also combat those isolates resistant to $\mathbf{1}$ alone. The use of the combination may also reduce the emergence of resistant isolates during treatments, due to the multiple antimicrobial targets of duel drug therapy and ease the selective pressure produced by broad-spectrum antibiotics. Further work should focus on triple combinations with enhancers, such as EGCG, to increase the potency of the combinations' antimicrobial action.

\section{EXPERIMENTAL SECTION}

Bacterial Strains and Media. Bacterial-type strains were purchased from the National Collection of Type Cultures (NCTC), Public Health England (Colindale, UK). The S. aureus (VISA) stain was obtained from the Network for Antimicrobial Resistance in $S$. aureus (NARSA, USA). Clinical isolates were sourced from Barts Health NHS Trust or the existing collection held at Queen Mary University London. Identification and routine antibiograms for clinical isolates were performed according to the laboratories accredited standard protocol (CPA). Curcumin powder ( $\geq 95 \%$ purity, cat. no. sc 294110, lot no. 191793-67) was purchased from Santa Cruz Biotechnology Inc. (Heidelberg, Germany). Polymyxin B sulfate powder was purchased from VWR International LTD (Leighton Buzzard, UK). All media was purchased from Thermo Fisher Inc. (Basingstoke, UK) and autoclaved prior to use.

Antimicrobial Susceptibility Assays. Minimum inhibitory concentrations of $\mathbf{1}$ and $\mathbf{2}$ were determined alone and in combination against 23 clinical isolates and type strains of Gram-negative and Gram-positive bacteria, related to traumatic wound infections, with varying degrees of antibiotic resistance (Table 1). Checkerboard assays, performed in separate 96-well microtiter plates, were inoculated with IsoSensitest broth containing $10^{6}$ colony-forming units $/ \mathrm{mL}$ of each isolate. Checkerboard assays were read after $24 \mathrm{~h}$ of incubation at $37{ }^{\circ} \mathrm{C}$. Fractional inhibitory concentration indices were calculated based on the method previously described, ${ }^{32}$ whereby FICa $=$ MIC of compound $\mathrm{a}+$ compound $\mathrm{b} / \mathrm{MIC}$ of compound $\mathrm{a}, \mathrm{FICb}=\mathrm{MIC}$ of compound $\mathrm{b}+$ compound $\mathrm{a} / \mathrm{MIC}$ of compound $\mathrm{b}$, and FICs $=$ FICa + FICb. FICIs $\leq 0.5$ were recorded as synergistic, values $>0.5-4.0$ were recorded as an additive effect, and a value $>0.4$ was considered antagonistic. Where the MIC was not attained, the dilution above the maximum dose was used to calculate the FICI. All experiments were carried out in triplicate, and results are presented as mean values.

Time-kill assays were undertaken to determine the antibacterial activity of mono- and combination therapies against $A$. baumannii (ATCC 19606), P. aeruginosa (ATCC 27853), methicillin-resistant $S$. aureus (NCTC 12493), and Stenotrophomonas maltophilia (NCTC 10258 ) over $24 \mathrm{~h}$. A 1/1000 dilution of an overnight culture (16 h in Mueller-Hinton 2 broth) (approximately $10^{6} \mathrm{CFU} / \mathrm{mL}$ ) was used as the starting inoculum ( $10 \mathrm{~mL}$ in universal tubes), before the addition of 1 (×1 MIC), 2 ( $\times 1 \mathrm{MIC})$, or combinations ( $\times 1$ MIC 1: $\times 0.5$ MIC 2). Cultures were incubated at $37^{\circ} \mathrm{C}$ under continuous agitation for $24 \mathrm{~h}$. At time intervals of $0,2,4,6$, and $24 \mathrm{~h}$ postinoculation, $100 \mu \mathrm{L}$ samples were collected, serially diluted, and plated onto IsoSensitest agar. Inoculated plates were incubated at $37{ }^{\circ} \mathrm{C}$ for $20 \mathrm{~h}$ before colonies were counted. Time-kill curves ( $\mathrm{CFU} / \mathrm{mL}$ vs time) were plotted using GraphPad software. Synergy was defined as bactericidal activity $\left(\geq 2 \log _{10}\right.$ difference in $\left.\mathrm{CFU} / \mathrm{mL}\right)$ of the combination compared to the single agent after $24 \mathrm{~h}$ of incubation.

Skin Cell Toxicity Assays. Cytotoxicity assays, versus human skin cells, were undertaken to assess the potential of $1 / 2$ combinations as topical treatments for skin infections. Skin cell toxicity assays were performed using the CytoTox 96 nonradioactive cytotoxicity assay (Promega Co., Southampton, UK) to monitor lactate dehydrogenase $(\mathrm{LDH})$, an indicator of percent cell toxicity. Human epidermal keratinocytes $(\mathrm{HaCaT})$ were plated at 10000 cells per well in 96-well plates and grown until subconfluent, in phenol-free Dulbecco's modified Eagle's medium supplemented with $2.5 \%(\mathrm{v} / \mathrm{v})$ fetal bovine serum (FBS) and $2 \mathrm{mM}$ L-glutamine. Double dilutions of 1, 2, and combinations of both agents were performed in vertical wells on a 96well microtiter plate before being transferred to a 96-well microtiter plate with half the plate containing human keratinocytes and the second half containing just $2 \%$ FBS phenol-free media. Final concentrations were 25-0 $\mu \mathrm{M}$ for 1 and $174-0 \mu \mathrm{M}$ for 2 . Concentrations of the combinations were the sum of $\mathbf{1}$ and 2 for monotherapy (2:1). DMSO, phosphate-buffered saline (PBS), and $\mathrm{LDH}$ positive controls were added to the plate. Microtiter plates were incubated at $37^{\circ} \mathrm{C}$ for $4 \mathrm{~h}$. After this time cells were washed in PBS. To each well was added $10 \mu \mathrm{L}$ of lysis solution, and the mixture incubated at $37{ }^{\circ} \mathrm{C}$ for $40 \mathrm{~min}$ before centrifugation at $250 \mathrm{~g}$ for $4 \mathrm{~min}$. From each well, $50 \mu \mathrm{L}$ of the supernatant was added to a sterile flatbottom enzymatic assay plate, before $50 \mu \mathrm{L}$ of reconstituted substrate mix was added to each well and incubated at room temperature, protected from light, for $30 \mathrm{~min}$. After incubation, $50 \mu \mathrm{L}$ of stop solution was added to each well, and absorbance was recorded using a plate reader (Wallac 1420 VICOTOR2, PerkinElmer, USA) set at 490 $\mathrm{nm}$. All assays were performed in duplicate. Percentage cytotoxicity was calculated, and graphs were plotted using Excel 2013.

Statistical Analysis. Student $t$ tests were performed using Excel 2013, to check for any significant differences between data sets.

\section{AUTHOR INFORMATION}

\section{Corresponding Author}

*Tel: +44 1483 683882. E-mail: jono.betts@surrey.ac.uk.

Notes

The authors declare no competing financial interest.

\section{ACKNOWLEDGMENTS}

We gratefully acknowledge the Barts Health NHS Trust, London, UK, for clinical samples.

\section{REFERENCES}

(1) Dryden, M. S. J. Antimicrob. Chemother. 2010, 65, iii35-iii44.

(2) Cosgrove, S. E. Clin. Infect. Dis. 2006, 42, S82-S89.

(3) Ouderkirk, J. P.; Nord, J. A.; Turett, G. S.; Kislak, J. W. Antimicrob. Agents Chemother. 2003, 47, 2659-2662.

(4) Tangden, T.; Giske, C. G. J. Intern. Med. 2015, 277, 501-512.

(5) Maragakis, L. L.; Perl, T. M. Clin. Infect. Dis. 2008, 46, 12541263.

(6) Godebo, G.; Kibra, G.; Tassew, H. Ann. Clin. Microbiol. Antimicrob. 2013, 12, 17.

(7) Pivanesea, H.; Balasoiu, M.; Ciurea, M. E.; Bakasoiu, A. T.; Manescu, R. Chirurgia 2014, 109, 73-79.

(8) Worthington, R. J.; Melander, C. Trends Biotechnol. 2013, 31, 177-184.

(9) Panaccio, V. Can. Med. Assoc. J. 1956, 75, 592-593.

(10) van Saene, J. J. M.; van Saene, H. K. F.; Tarko-Smit, N. J.; Beukeveid, G. J. Epidemiol. Infect. 1988, 100, 407-417.

(11) Nordmann, P.; Naas, T.; Poirel, L. Emerging Infect. Dis. 2011, 17, $1791-1798$.

(12) Falagas, M. E.; Rafailidis, P. I.; Matthaiou, D. K. Drug Resist. Updates 2010, 13, 123-138. 
(13) Liu, Y. Y.; Wang, Y.; Walsh, T. R.; Yi, L. X.; Zhang, R.; Spencer, J.; Doi, Y.; Tian, G.; Dong, B.; Huang, X.; Yu, L. F.; Gu, D.; Ren, H.; Chen, X.; Lu, L.; He, D.; Zhou, H.; Liang, Z.; Liu, J. H.; Shen, J. Lancet Infect. Dis. 2015, 16, 161-168.

(14) De, R.; Kundu, P.; Swarnakar, S.; Ramamurthy, T.; Chowdhury, A.; Nair, G. B.; Mukhopadyay, A. K. Antimicrob. Agents Chemother. 2009, 53, 1592-1597.

(15) Hu, P.; Huang, P.; Chen, M. W. Arch. Oral Biol. 2013, 58, $1343-1348$.

(16) Phan, T. T.; See, P.; Lee, S. T.; Chan, S. Y. J. Trauma 2001, 51, 927-931.

(17) Huang, M. T.; Yen, P.; Xie, J. G.; Han, J.; Frenkel, K.; Grunberger, D.; Conney, A. H. Carcinogenesis 1997, 18, 83-88.

(18) Betts, J. W.; Wareham, D. W. BMC Microbiol. 2014, 14, 172.

(19) Mihu, M. R.; Martinez, L. R. Virulence 2011, 2, 97-102.

(20) Rai, D.; Singh, J. K.; Roy, N.; Panda, D. Biochem. J. 2008, 410, 147-155.

(21) Park, B. S.; Kim, J. G.; Kim, M. R.; Lee, S. E.; Takeoka, G. R.; Oh, K. B.; Kim, J. H. J. Agric. Food Chem. 2005, 53, 9005-9009.

(22) Bhawana; Basniwal, R. K.; Buttar, H. S.; Jain, V. K.; Jain, N. J. Agric. Food Chem. 2011, 59, 2056-2061.

(23) Mun, S. H.; Kim, S. B.; Kong, R.; Choi, J. G.; Kin, Y. C.; Shin, D. W.; Kang, O. H.; Kwon, D. Y. Molecules 2014, 19, 18283-18295.

(24) Velkov, T.; Thompson, P. E.; Nation, R. L.; Li, J. J. Med. Chem. 2010, 53, 1898-1916.

(25) Zhao, R.; Yang, B.; Wang, L.; Xue, P.; Deng, B.; Zhang, G.; Jiang, S.; Zhang, M.; Liu, M.; Pi, J.; Dawei, G. Oxid. Med. Cell. Longevity 2013, 2013, 412576.

(26) Natarajan, V. T.; Singh, A.; Kumar, A. A.; Sharma, P.; Kar, H. K.; Marriot, L.; Meunier, J. R.; Natarajan, K.; Rani, R.; Gokhale, R. S. J. Invest. Dermatol. 2010, 130, 2781-2789.

(27) Patel, N. A.; Patel, N. J.; Patel, R. P. Pharm. Dev. Technol. 2009, $14,83-92$.

(28) Cheng, A. L.; Hsu, C. H.; Lin, J. K.; Hsu, M. M.; Ho, Y. F.; Shen, T. S.; Ko, J. Y.; Lin, J. T.; et al. Anticancer Res. 2001, 21, 28952900.

(29) Sharma, R. A.; Euden, S. A.; Platton, S. L.; Cooke, D. N.; Shafayat, A.; Hewitt, H. R.; Marczylo, T. H.; Morgan, B.; et al. Clin. Cancer Res. 2004, 10, 6847-6854.

(30) Afshariani, R.; Farhadi, P.; Ghaffarpasand, F.; Roozbeth, J. Oman Med. J. 2014, 29, 330-334.

(31) Fang, J.-Y.; Hung, C.-H.; Chiu, H.-C.; Wang, J.-J.; Chan, T.-F. J. Pharm. Pharmacol. 2003, 55, 593-601.

(32) Hall, M. J.; Middleton, R. F.; Westmacott, D. J. Antimicrob. Chemother. 1983, 11, 427-433. 